\title{
Synthesis and Biological Activity of Chalcone Derivatives as Anti - Asthmatics Agents
}

\author{
MANUVESH SANGWAN ${ }^{1,2}$ and D P. PATHAK ${ }^{1,3}$ \\ ${ }^{1}$ Guru Jambheshwar University of Science and Technology, Hisar, India \\ ${ }^{2}$ New York State Department of Health, Albany, New York 12202 \\ ${ }^{3}$ Delhi Institute of Pharmaceutical Sciences and Research, New Delhi, 110017, India \\ manuvesh@gmail.com
}

Received 2 March 2016 / Accepted 19 March 2016

\begin{abstract}
The objective of this study was to explore novel compounds with benzylideneactophenone moiety, which have potential for anti-asthmatic activity and can be potential candidates for PDE4 inhibitory activity. Seventeen different substituted benzylidene acetophenone and chalcones derivatives have been synthesized by condensing derivatives of benzaldehyde and acetophenone using Claisen Schmidt condensation. All the compounds were purified and have been characterized by FTIR and NMR spectroscopy. Among 17 compounds, three compounds were selected for investigation for antiasthmatic activity. The anti-inflammatory and bronchodilatory activity of the novel products was evaluated by rat paw edema method and tracheal relaxation model. Among these compounds, compound 8 ((2E)-1-(3,4-dichlorophenyl)-3-(3,4-dimethoxyphenyl)-2-propen-1-one ) showed relatively higher anti-inflammatory activity which can be attributed to the substitution of hydrophobic group and 3, 4-dialkoxy groups, compound 8 showed a 59.8\% bronchodilatory response when compared to Rolipram (100\% response), whereas compounds 17 ((2E)-3-(2-Furyl)-1-(3hydroxyphenyl)-2-propen-1-one) and $\mathbf{1 3}$ ((2E)-3-(4-Ethylphenyl)-1-phenyl-2-propen-1-one) showed marginal activity i.e. $41.43 \%$ and $24.35 \%$ of the standard drug.
\end{abstract}

Keywords: Benzylideneacetophenone, Chalcone derivatives, Anti-asthmatic activity, PDE4 inhibitors

\section{Introduction}

Benzylideneacetophenone or chalcone derivatives have long been explored for their different pharmacological activities ${ }^{1-8}$. These compounds are originated from nature and can be easily synthesized in lab. It consist of open chain flavonoids in which the two aromatic rings are joined by a three carbon $\alpha . \beta$ unsaturated carbonyl system. The presence of reactive $\alpha, \beta$ unsaturated keto moiety in chalcones is considered to be mainly responsible for their pharmacological activity. In recent years, a variety of benzyldiene acetophenone derivatives have been explored for their biological activities which include antidiabetic, antifungal, anticancer, antiviral, insecticidal and enzyme inhibitory properties. In these studies, the presence of hydroxy, alkoxy, halogen groups in different position have been reported to 
possess wide range of biological and medicinal activities ${ }^{9-14}$. Recently, there has been a growing interest for the therapeutic applications of phosphodiesterase 4 (PDE4) inhibitors. Two main reasons for the basis of the rapid development of biochemical, medicinal and pharmacological research in this field of PDE4 inhibitors are; 1) there is a general conviction that PDE4 inhibitors have both anti-inflammatory and bronchodilatory activity ${ }^{9}$ and 2) new and promising therapeutic applications of PDE4 inhibitors in certain autoimmune disease e.g. rheumatoid arthritis, multiple sclerosis and type- 2 diabetes. Rolipram ${ }^{\circledR}$ and Enoximone ${ }^{\circledR}$ are the drugs of interest for our research. Among these drugs, the catechol ether moiety is responsible for the PDE4 inhibitory activity of these molecules. Enoximone, a PDE3/PDE4 inhibitor which has the benzylidene acetophenone like moiety. Although, these drugs promised to be better anti-asthmatic property but most of PDE4 inhibitors has been hindered by their potential emetic effects, which considered to be related to their inhibition of PDE4D receptors ${ }^{9-11}$. The structure features of PDE4 inhibitors and its similarity with chalcone derivatives motivated us to synthesize the benzylideneacetophenone derivatives mainly having 3, 4-dialkoxy substitution ${ }^{14-19}$.

\section{Experimental}

The appropriately substituted acetophenones and aldehydes were either synthesized in the lab or procured from the Sigma Aldrich. The standard drug Rolipram has been obtained from AG Scientific as a gift.

\section{Synthesis}

Benzylideneacetophenone derivatives were synthesized by using base catalyzed $(\mathrm{NaOH}$ or $\mathrm{KOH})$ Claisen-Schmidt condensation reaction ${ }^{17-19}$. The appropriately substituted acetophenones and aldehydes were either synthesized in the lab or procured from the Sigma Aldrich. Although, the detail description of the synthesis can be found in literature only brief description is provided here. A mixture of benzaldehyde derivatives and acetophenone derivatives $(1: 1)$ was dissolved in sufficient quantity of ethanol in a round-bottomed flask equipped with a magnetic stirrer. Then the saturated solution of $\mathrm{NaOH}\left(1 \mathrm{~g}\right.$ in $\left.10 \mathrm{~mL} \mathrm{H}_{2} \mathrm{O}\right)$ was added drop wise to the reaction mixture with stirring for 1 $\mathrm{h}$ or until the reaction gets complete. The mixture kept to reflux under vigorous stirring for three hours. The progress of the reaction was monitored by TLC using silica gel-G plates in n-hexane acetone mixture (3:1) and spots were monitored in iodine chamber. After reaction completion, solution mixture was quickly poured in the filtration assembly into the container which was kept at about $10-15{ }^{\circ} \mathrm{C}$ to precipitate the solids. Crystalline compound in yellow to orange color were obtained. After filtering off, the crude products were dried in air and recrystallized by ethanol and characterized. The scheme of reaction is depicted in Figure 1.

The residue which were not easily purified (compound 8,9,16,17) were purified on column chromatography (silica gel with $15 \%$ ethyl acetate in hexane) to obtain pure products (Figure 1).<smiles>O=Cc1ccccc1</smiles>

Acetophenone<smiles>O=Cc1ccccc1</smiles>

Benzaldehyde
1) with $\mathrm{KOH} / \mathrm{NaOH}$ 2) Refluxed 3 hours<smiles>O=C(COC(=O)O[Ga]OC(=O)c1ccccc1)OOc1ccccc1</smiles> 
<smiles>[R6]c1ccc(/C=C/C(=O)c2cc([R])c([R2])cc2[R9])cc1[R4]</smiles>

Structure 1<smiles>[R]c1ccc([R2])c(C(=O)/C=C/c2ccco2)c1</smiles>

Structure 2

Figure 1. Experimental scheme of synthesis of compounds (Structure 1 represents design of compounds based on benzylidene acetophenone skeleton (Table 1), Structure 2 indicates, 2furyl)-1-(3-hydroxyphenyl)-2-propen-1-one derivatives (Table 2)).

\section{Results and Discussion}

The melting points of the compounds were determined in B-540 Buchi melting-point apparatus and are uncorrected. The characterizations of compounds were performed by FTIR, melting point check and NMR spectra. FTIR spectra were recorded on a Perkin Elmer 1800(FTIR) spectrometer and UV-Vis spectra of compounds were measured in Perkin Elmer double beam UV spectrophotometer. Additionally, all compounds were characterized by ${ }^{1} \mathrm{H}$ NMR (Brooks Instrument DMX400MHz) spectra with TMS as an internal standard and chemical shifts are measured in ppm interpreted with the structural features. The derivatives of the parent reactant molecules benzaldehyde and acetophenone include a variation of functional groups at $\mathrm{R}, \mathrm{R} 1$ and $\mathrm{R} 2$ position, such as methyl $\left(-\mathrm{CH}_{3}\right)$, methoxy $\left(-\mathrm{OCH}_{3}\right)$, chloro $(-\mathrm{Cl})$, ethyl $\left(-\mathrm{CH}_{3} \mathrm{CH}_{3}\right)$ and hydroxy $(-\mathrm{OH})$ etc and in various positions around the phenyl ring (Table $1 \& 2$ ).

Table 1. Derivatives of benzylidene acetophenone

\begin{tabular}{cccccc}
\hline Compound & $\mathrm{R} 1$ & $\mathrm{R} 2$ & $\mathrm{R} 3$ & $\mathrm{R} 4$ & $\mathrm{R} 5$ \\
\hline 1 & $\mathrm{H}$ & $\mathrm{H}$ & $\mathrm{H}$ & $\mathrm{H}$ & $\mathrm{H}$ \\
2 & $\mathrm{H}$ & $\mathrm{H}$ & $\mathrm{H}$ & $\mathrm{OCH}_{3}$ & $\mathrm{OCH}_{3}$ \\
3 & $\mathrm{H}$ & $\mathrm{OCH}$ & $\mathrm{H}$ & $\mathrm{OCH}_{3}$ & $\mathrm{OCH}_{3}$ \\
4 & $\mathrm{H}$ & $\mathrm{Br}$ & $\mathrm{H}$ & $\mathrm{OCH}_{3}$ & $\mathrm{OCH}_{3}$ \\
5 & $\mathrm{H}$ & $\mathrm{NH}_{2}$ & $\mathrm{H}$ & $\mathrm{OCH}_{3}$ & $\mathrm{OCH}_{3}$ \\
6 & $\mathrm{H}$ & $\mathrm{Cl}$ & $\mathrm{H}$ & $\mathrm{OCH}_{3}$ & $\mathrm{OCH}_{3}$ \\
7 & $\mathrm{H}$ & $\mathrm{OH}$ & $\mathrm{H}$ & $\mathrm{OCH}_{3}$ & $\mathrm{OCH}_{3}$ \\
8 & $\mathrm{Cl}$ & $\mathrm{Cl}$ & $\mathrm{H}$ & $\mathrm{OCH}_{3}$ & $\mathrm{OCH}_{3}$ \\
9 & $\mathrm{H}$ & $\mathrm{H}$ & $\mathrm{OH}$ & $\mathrm{OCH}_{3}$ & $\mathrm{OCH}_{3}$ \\
10 & $\mathrm{H}$ & $\mathrm{H}$ & $\mathrm{H}$ & $\mathrm{H}$ & $\mathrm{Cl}$ \\
11 & $\mathrm{Cl}$ & $\mathrm{H}$ & $\mathrm{H}$ & $\mathrm{H}$ & $\mathrm{Cl}$ \\
12 & $\mathrm{Cl}$ & $\mathrm{H}$ & $\mathrm{H}$ & $\mathrm{H}$ & $\mathrm{OCH}_{3}$ \\
13 & $\mathrm{H}$ & $\mathrm{H}$ & $\mathrm{H}$ & $\mathrm{H}$ & $\mathrm{CH}_{3} \mathrm{CH}_{3}$ \\
14 & $\mathrm{Cl}$ & $\mathrm{OH}$ & $\mathrm{H}$ & $\mathrm{H}$ & $\mathrm{OCH}_{3}$ \\
15 & $\mathrm{Cl}$ & $\mathrm{Cl}$ & & $\mathrm{H}$ & $\mathrm{OH}^{2}$ \\
\hline
\end{tabular}

Table 2. Derivatives of 2-Furyl)-1-(3-hydroxyphenyl)-2-propen-1-one

\begin{tabular}{ccc}
\hline Compound & $\mathrm{R} 1$ & $\mathrm{R} 2$ \\
\hline 16 & $\mathrm{Cl}$ & $\mathrm{OH}$ \\
17 & $\mathrm{OH}$ & $\mathrm{H}$ \\
\hline
\end{tabular}


In the NMR spectra, there are various factors which can complicate the interpretation of NMR spectra which include hydroxyl group and the presence of intermolecular hydrogen bonding. NMR spectra of these compounds are expected to influence by temperature, concentration and solvent polarity, which complicates its interpretation. Similarly, the spectrum of the compounds with amino group is somewhat complicated due to spin coupling of the proton with the nitrogen nucleus, and the variable rate of exchange of the labile amino proton. Although, halogen substitution does not introduce a new peak in a proton NMR spectrum but it can affect peak positions due to its pulling effect and also the splitting patterns on the aromatic protons can be affected. Following is the chemical profile of all the 17 compounds synthesized

Compound 1: (2E)-1,3-Diphenyl-2-propen-1-one

M.pt 55-57 ${ }^{\circ} \mathrm{C}, \mathrm{MW}=208.25$, IR (KBr): 3600-2750 (OH Str.), 3060-3020 $\mathrm{cm}^{-1}$ (Aromatic, $\mathrm{C}-\mathrm{H}$ str.), $2800 \mathrm{~cm}^{-1} \mathrm{CH}$ stretching, $1760 \mathrm{~cm}^{-1}$ (Carboxylic, C-O str.), $746 \mathrm{~cm}^{-1}{ }^{1} \mathrm{H}$ NMR $\left(\mathrm{CDCl}_{3}\right), \delta(\mathrm{ppm}): 7.85\left(\mathrm{~d}, 2 \mathrm{H}, \mathrm{Ar} 3^{\prime}, 5^{\prime} \mathrm{H}\right), 7.87\left(\mathrm{~d}, 2 \mathrm{H}, \mathrm{Ar} 2^{\prime}, 6^{\prime} \mathrm{H}\right), 7.58(\mathrm{~d}, 1 \mathrm{Ha}, \mathrm{J}=16 \mathrm{~Hz}$, $=\mathrm{CH}), 7.61(\mathrm{~d}, 1 \mathrm{Hb}, \mathrm{J}=16 \mathrm{~Hz},=\mathrm{CH}), 6.92(\mathrm{~d}, 2 \mathrm{H}, \mathrm{Ar} 2 ", 6 "-\mathrm{H}), 6.94(\mathrm{~d}, 2 \mathrm{H}, \mathrm{Ar} 3 ", 5 "-\mathrm{H})$.

Compound 2:(2E)-3-(3,4-Dimethoxyphenyl)-1-phenyl-2-propen-1-one

M.pt 88-89 ${ }^{\circ} \mathrm{C}, \mathrm{MW}=267.30, \mathrm{IR}(\mathrm{KBr}): 1658(>\mathrm{C}=\mathrm{O}$ in conjugation with $\mathrm{C}=\mathrm{C}), 1596$, 1540 $(>\mathrm{C}=\mathrm{C}<$ in conjugation with $\mathrm{C}=\mathrm{O}){ }^{1} \mathrm{HNMR}\left(\mathrm{CDCl}_{3}\right), \delta(\mathrm{ppm}): 7.82\left(\mathrm{~d}, 2 \mathrm{H}, \mathrm{Ar} 3{ }^{\prime}, 5^{\prime} \mathrm{H}\right)$, $7.87(\mathrm{~d}, 2 \mathrm{H}$, Ar 2',6'H), 7.58(d,1Ha, J = $16 \mathrm{~Hz},=\mathrm{CH}), 7.41(\mathrm{~d}, 1 \mathrm{Hb}, \mathrm{J}=16 \mathrm{~Hz},=\mathrm{CH}), 6.92(\mathrm{~d}$, 2H, Ar 2",6"-H), 6.84(d,2H, Ar 3", 5"- H), 3.81(s,3H, Ar 4"-OCH3).

Compound 3: (2E)-3-(3,4-Dimethoxyphenyl)-1-(4-methoxyphenyl)-2-propen-1-one

M.pt 88-89 ${ }^{\circ} \mathrm{C}, \quad \mathrm{MW}=298.30$, IR (KBr): 1658 ( $>\mathrm{C}=\mathrm{O}$ in conjugation with $\mathrm{C}=\mathrm{C}$ ), $1596,1540(>\mathrm{C}=\mathrm{C}<$ in conjugation with $\mathrm{C}=\mathrm{O}) 1760 \mathrm{~cm}^{-1}$ (Carboxylic, C-O str.) ${ }^{1} \mathrm{HNMR}$ $\left(\mathrm{CDCl}_{3}\right), \delta(\mathrm{ppm}): 7.72\left(\mathrm{~d}, 2 \mathrm{H}, \mathrm{Ar} 3^{\prime}, 5^{\prime} \mathrm{H}\right), 7.67\left(\mathrm{~d}, 2 \mathrm{H}, \mathrm{Ar} 2^{\prime}, 66^{\prime} \mathrm{H}\right), 7.58(\mathrm{~d}, 1 \mathrm{Ha}, \mathrm{J}=16 \mathrm{~Hz}$, $=\mathrm{CH}), 7.41(\mathrm{~d}, 1 \mathrm{Hb}, \mathrm{J}=16 \mathrm{~Hz},=\mathrm{CH}), 6.82(\mathrm{~d}, 2 \mathrm{H}$, Ar 2",6"-H), 6.94(d,2H, Ar 3", 5"- H), $3.71\left(\mathrm{~s}, 3 \mathrm{H}, \mathrm{Ar} 4 "-\mathrm{OCH}_{3}\right)$.

Compound 4: (2E)-1-(4-Bromophenyl)-3-(3,4-dimethoxyphenyl)-2-propen-1-one

M.pt 158-160 ${ }^{\circ} \mathrm{C}, \mathrm{MW}=347.03$, IR (KBr): C-Br stretch 690-515 cm $3060-3020 \mathrm{~cm}^{-1}$ (Aromatic, C-H str.), $1760 \mathrm{~cm}^{-1}$ (Carboxylic, C-O str.), $746 \mathrm{~cm}^{-1}{ }^{1} \mathrm{H} \mathrm{NMR}\left(\mathrm{CDCl}_{3}\right), \delta$ (ppm): 7.65(d, 2H, Ar 3', 5'H), 7.87 (d, 2H, Ar 2',6'H), 7.58(d,1Ha, J = 14 Hz, =CH), 7.61(d, 1 Hb, J $=15 \mathrm{~Hz},=\mathrm{CH}), 6.72$ (d, 2H, Ar 2",6"-H), 6.74(d,2H, Ar 3", 5"- H).

Compound 5: (2E)-1-(4-Aminophenyl)-3-(3,4-dimethoxyphenyl)-2-propen-1-one

M.pt $188-189^{\circ} \mathrm{C}, \mathrm{MW}=283.22$, IR $(\mathrm{KBr}): 1281,761(\mathrm{NH}$ and $\mathrm{C}-\mathrm{N}$ stretch) $1658(>\mathrm{C}=\mathrm{O}$ in conjugation with $\mathrm{C}=\mathrm{C}), \quad 1596,1540(>\mathrm{C}=\mathrm{C}<$ in conjugation with $\mathrm{C}=\mathrm{O}) 1760 \mathrm{~cm}^{-1}$ (Carboxylic, C-O str.) ${ }^{1} \mathrm{HNMR}\left(\mathrm{CDCl}_{3}\right), \delta(\mathrm{ppm}): 7.72\left(\mathrm{~d}, 2 \mathrm{H}, \mathrm{Ar} 3^{\prime}, 5^{\prime} \mathrm{H}\right), 7.47(\mathrm{~d}, 2 \mathrm{H}, \mathrm{Ar}$ $\left.2^{\prime}, 6^{\prime} \mathrm{H}\right), 7.58(\mathrm{~d}, 1 \mathrm{Ha}, \mathrm{J}=18 \mathrm{~Hz},=\mathrm{CH}), 7.61(\mathrm{~d}, 1 \mathrm{Hb}, \mathrm{J}=16 \mathrm{~Hz},=\mathrm{CH}), 6.96(\mathrm{~d}, 2 \mathrm{H}, \mathrm{Ar}$ 2",6"-H), 6.84(d,2H, Ar 3", 5"- H), 3.61(s,3H, Ar 4"-- $\left.\mathrm{OCH}_{3}\right)$.

Compound 6: 4'-Chloro-3,4-dimethoxychalcone:

M.pt 143-149 ${ }^{\circ} \mathrm{C}, \mathrm{MW}=302.75$, IR $(\mathrm{KBr}): 1281,741 \quad(\mathrm{C}-\mathrm{Cl}$ strestch $) 1657 \quad(>\mathrm{C}=\mathrm{O}$ in conjugation with $\mathrm{C}=\mathrm{C}), 1594,1540(>\mathrm{C}=\mathrm{C}<$ in conjugation with $\mathrm{C}=\mathrm{O}) 1760 \mathrm{~cm}^{-1}$ (Carboxylic, C-O str.) ${ }^{1} \mathrm{HNMR}\left(\mathrm{CDCl}_{3}\right), \delta(\mathrm{ppm}): 7.72\left(\mathrm{~d}, 2 \mathrm{H}, \mathrm{Ar} 3^{\prime}, 5^{\prime} \mathrm{H}\right), 7.47(\mathrm{~d}, 2 \mathrm{H}, \mathrm{Ar}$ $\left.2^{\prime}, 6 ' \mathrm{H}\right), 7.58(\mathrm{~d}, 1 \mathrm{Ha}, \mathrm{J}=18 \mathrm{~Hz},=\mathrm{CH}), 7.61(\mathrm{~d}, 1 \mathrm{Hb}, \mathrm{J}=16 \mathrm{~Hz},=\mathrm{CH}), 6.96(\mathrm{~d}, 2 \mathrm{H}, \mathrm{Ar}$ 2",6"-H), 6.84(d,2H, Ar 3", 5"- H), 3.61(s,3H, Ar 4"- $\left.\mathrm{OCH}_{3}\right)$. 
Compound 7: (2E)-3-(3,4-Dimethoxyphenyl)-1-(4-hydroxyphenyl)-2-propen-1-one

M.pt 159-163 ${ }^{\circ} \mathrm{C}, \mathrm{MW}=284.34$, IR (KBr): 1280, 3012 (O-H strestch) $1657(>\mathrm{C}=\mathrm{O}$ in conjugation with $\mathrm{C}=\mathrm{C}), 1594,1540(>\mathrm{C}=\mathrm{C}<$ in conjugation with $\mathrm{C}=\mathrm{O}) 1760 \mathrm{~cm}^{-1}$ (Carboxylic, C-O str.) ${ }^{1} \mathrm{HNMR}\left(\mathrm{CDCl}_{3}\right), \delta(\mathrm{ppm}): 7.72\left(\mathrm{~d}, 2 \mathrm{H}, \mathrm{Ar} 3^{\prime}, 5^{\prime} \mathrm{H}\right), 7.47(\mathrm{~d}, 2 \mathrm{H}, \mathrm{Ar}$ $\left.2^{\prime}, 6^{\prime} \mathrm{H}\right), 7.58(\mathrm{~d}, 1 \mathrm{Ha}, \mathrm{J}=18 \mathrm{~Hz},=\mathrm{CH}), 7.61(\mathrm{~d}, 1 \mathrm{Hb}, \mathrm{J}=16 \mathrm{~Hz},=\mathrm{CH}), 6.96$ (d, 2H, Ar 2",6"-H), 6.94(d,2H, Ar 3", 5"- H), 3.61(s,3H, Ar 4"- $\left.\mathrm{OCH}_{3}\right)$.

Compound 8: (2E)-1-(3,4-Dichlorophenyl)-3-(3,4-dimethoxyphenyl)-2-propen-1-one

M.pt 155-157 ${ }^{\circ} \mathrm{C}, \mathrm{MW}=336.34$, IR $(\mathrm{KBr}): 1281,741(\mathrm{C}-\mathrm{Cl}$ stretch) 1657 ( $>\mathrm{C}=\mathrm{O}$ in conjugation with $\mathrm{C}=\mathrm{C}), 1594,1540(>\mathrm{C}=\mathrm{C}<$ in conjugation with $\mathrm{C}=\mathrm{O}) 1760 \mathrm{~cm}^{-1}$ (Carboxylic, C-O str.) ${ }^{1} \mathrm{HNMR}\left(\mathrm{CDCl}_{3}\right), \delta(\mathrm{ppm}):$ 7.72(d, 2H, Ar 3', 5'H), 7.47 (d, 2H, Ar $\left.2^{\prime}, 6^{\prime} \mathrm{H}\right), 7.58(\mathrm{~d}, 1 \mathrm{Ha}, \mathrm{J}=18 \mathrm{~Hz},=\mathrm{CH}), 7.61(\mathrm{~d}, 1 \mathrm{Hb}, \mathrm{J}=16 \mathrm{~Hz},=\mathrm{CH}), 6.96(\mathrm{~d}, 2 \mathrm{H}, \mathrm{Ar}$ 2",6"-H), 6.94(d,2H, Ar 3", 5"- H), 3.61(s,3H, Ar 4"- $\left.\mathrm{OCH}_{3}\right)$.

Compound 9: H9 2'-Hydroxy-3,4-dimethoxychalcone

M.pt $160-163{ }^{\circ} \mathrm{C}, \mathrm{MW}=284.34$, IR $(\mathrm{KBr}): 1280,3012(\mathrm{O}-\mathrm{H}$ strestch) $1657(>\mathrm{C}=\mathrm{O}$ in conjugation with $\mathrm{C}=\mathrm{C}), 1594,1540(>\mathrm{C}=\mathrm{C}<$ in conjugation with $\mathrm{C}=\mathrm{O}) 1760 \mathrm{~cm}^{-1}$ (Carboxylic, C-O str.) ${ }^{1} \mathrm{HNMR}\left(\mathrm{CDCl}_{3}\right), \delta(\mathrm{ppm}):$ 7.72(d, 2H, Ar 3', 5'H), 7.47 (d, 2H, Ar $\left.2^{\prime}, 6 ' \mathrm{H}\right), 7.58(\mathrm{~d}, 1 \mathrm{Ha}, \mathrm{J}=18 \mathrm{~Hz},=\mathrm{CH}), 7.61(\mathrm{~d}, 1 \mathrm{Hb}, \mathrm{J}=16 \mathrm{~Hz},=\mathrm{CH}), 6.96(\mathrm{~d}, 2 \mathrm{H}, \mathrm{Ar}$ 2",6"-H), 6.94(d,2H, Ar 3", 5"- H), 3.61(s,3H, Ar 4"-OCH ${ }_{3}$.

Compound 10: 4-Chlorochalcone, (2E)-3-(4-Chlorophenyl)-1-phenyl-2-propen-1-one

M.pt 114-116 ${ }^{\circ} \mathrm{C}, \mathrm{MW}=242.7$, IR (KBr): 1281, 741 (C-Cl strestch)1657 (>C=O in conjugation with $\mathrm{C}=\mathrm{C}), 1594,1540(>\mathrm{C}=\mathrm{C}<$ in conjugation with $\mathrm{C}=\mathrm{O}){ }^{1} \mathrm{HNMR}\left(\mathrm{CDCl}_{3}\right), \delta$ (ppm): 7.72(d, 2H, Ar 3', 5'H), 7.47 (d, 2H, Ar 2',6'H), 7.58(d,1Ha, J = 18 Hz, =CH), 7.61(d, $1 \mathrm{Hb}, \mathrm{J}=16 \mathrm{~Hz},=\mathrm{CH}), 6.96$ (d, 2H, Ar 2",6"-H), 6.94(d,2H, Ar 3", 5"- H).

Compound 11: 4',4-dichlorochalcone or (2E)-1,3-Bis(4-chlorophenyl)-2propen-1-one

M.pt 156-160 ${ }^{\circ} \mathrm{C}, \mathrm{MW}=277.17$, IR $(\mathrm{KBr}): 1281,741 \quad(\mathrm{C}-\mathrm{Cl}$ strestch $) 1657 \quad(>\mathrm{C}=\mathrm{O}$ in conjugation with $\mathrm{C}=\mathrm{C}), 1594,1540(>\mathrm{C}=\mathrm{C}<$ in conjugation with $\mathrm{C}=\mathrm{O}) 1 \mathrm{HNMR}(\mathrm{CDCl} 3), \delta$ (ppm): 7.72(d, 2H, Ar 3', 5'H), 7.47 (d, 2H, Ar 2',6'H), 7.58(d,1Ha, J = 18 Hz, =CH), 7.61(d, $1 \mathrm{Hb}, \mathrm{J}=16 \mathrm{~Hz},=\mathrm{CH}), 6.96$ (d, 2H, Ar 2",6"-H), 6.94(d,2H, Ar 3", 5"- H).

Compound 12: 4'-Chloro-4-methoxychalcone or (2E)-1-(4-Chlorophenyl)-3-(4methoxyphenyl)-2-propen-1-one

M.pt 127-129 ${ }^{\circ} \mathrm{C}, \mathrm{MW}=272.72$, IR (KBr): 1281, 741 (C-Cl strestch) 1280, 3012 (O-H stretch)1657 ( $>\mathrm{C}=\mathrm{O}$ in conjugation with $\mathrm{C}=\mathrm{C}), 1594,1540(>\mathrm{C}=\mathrm{C}<$ in conjugation with $\mathrm{C}=\mathrm{O})$ 1HNMR $(\mathrm{CDCl} 3), \delta(\mathrm{ppm}):$ 7.72(d, 2H, Ar 3', 5'H), $7.47(\mathrm{~d}, 2 \mathrm{H}$, Ar 2',6'H), $7.58(\mathrm{~d}, 1 \mathrm{Ha}, \mathrm{J}=18 \mathrm{~Hz},=\mathrm{CH}), 7.61(\mathrm{~d}, 1 \mathrm{Hb}, \mathrm{J}=16 \mathrm{~Hz},=\mathrm{CH}), 6.96(\mathrm{~d}, 2 \mathrm{H}$, Ar 2",6"-H), 6.94(d,2H, Ar 3", 5"- H.

Compound 13: (2E)-3-(4-Ethylphenyl)-1-phenyl-2-propen-1-one

M.pt 163-165 ${ }^{\circ} \mathrm{C}, \mathrm{MW}=236.38$, IR (KBr): 3281(C-CH stretch) 1280, 1657 ( $>\mathrm{C}=\mathrm{O}$ in conjugation with $\mathrm{C}=\mathrm{C}), 1594,1540(>\mathrm{C}=\mathrm{C}<$ in conjugation with $\mathrm{C}=\mathrm{O}){ }^{1} \mathrm{HNMR}\left(\mathrm{CDCl}_{3}\right), \delta$ (ppm): 7.72(d, 2H, Ar 3', 5'H), 7.47 (d, 2H, Ar 2',6'H), 7.58(d,1Ha, J = $18 \mathrm{~Hz},=\mathrm{CH}), 7.61(\mathrm{~d}$, $1 \mathrm{Hb}, \mathrm{J}=16 \mathrm{~Hz},=\mathrm{CH}), 6.96$ (d, 2H, Ar 2",6"-H), 6.94(d,2H, Ar 3", 5"- H. 
Compound 14: (2E)-1-(3-Chloro-4-hydroxyphenyl)-3-(4-methoxyphenyl)-2-

propen-1-one

M.pt 143-145 ${ }^{\circ} \mathrm{C}, \mathrm{MW}=288.72$, IR (KBr): 1281, 741 (C-Cl stretch) 3281(C-CH stretch) 1280, 1657 ( $>\mathrm{C}=\mathrm{O}$ in conjugation with $\mathrm{C}=\mathrm{C}), 1594,1540(>\mathrm{C}=\mathrm{C}<$ in conjugation with $\mathrm{C}=\mathrm{O}) 1 \mathrm{HNMR}$ $(\mathrm{CDCl} 3), \delta(\mathrm{ppm}): 7.72\left(\mathrm{~d}, 2 \mathrm{H}, \mathrm{Ar} 3^{\prime}, 5^{\prime} \mathrm{H}\right), 7.47$ (d, 2H, Ar 2',6'H), 7.58(d,1Ha, J = $18 \mathrm{~Hz}$, $=\mathrm{CH}), 7.61(\mathrm{~d}, 1 \mathrm{Hb}, \mathrm{J}=16 \mathrm{~Hz},=\mathrm{CH}), 6.96$ (d, 2H, Ar 2",6"-H), 6.94(d,2H, Ar 3", 5"- H.

Compound 15: (2E)-1-(3,4-Dichlorophenyl)-3-(4-hydroxyphenyl)-2-propen-1-one M.pt 153-155 ${ }^{\circ} \mathrm{C}, \mathrm{MW}=293.15$, IR (KBr): 1281, 741 (C-Cl stretch), 3281(C-CH stretch) 1280, $1657(>\mathrm{C}=\mathrm{O}$ in conjugation with $\mathrm{C}=\mathrm{C}), 1594,1540(>\mathrm{C}=\mathrm{C}<$ in conjugation with $\mathrm{C}=\mathrm{O})$, ${ }^{1} \mathrm{HNMR}\left(\mathrm{CDCl}_{3}\right), \delta(\mathrm{ppm}): 7.72\left(\mathrm{~d}, 2 \mathrm{H}, \mathrm{Ar} 3^{\prime}, 5^{\prime} \mathrm{H}\right), 7.17\left(\mathrm{~d}, 2 \mathrm{H}, \mathrm{Ar} 2^{\prime}, 6^{\prime} \mathrm{H}\right), 7.58(\mathrm{~d}, 1 \mathrm{Ha}, \mathrm{J}=18$ $\mathrm{Hz},=\mathrm{CH}), 7.61(\mathrm{~d}, 1 \mathrm{Hb}, \mathrm{J}=8.41 \mathrm{~Hz},=\mathrm{CH}), 6.96$ (d, 2H, Ar 2",6"-H), 6.94(d,2H, Ar 3", 5"- H.

Compound 16: (2E)-1-(5-Chloro-2-hydroxyphenyl)-3-(2-furyl)-2-propen-1-one M.pt $=135^{\circ} \mathrm{C}, \mathrm{MW}=248.66$, IR (KBr): 1281, 741 (C-Cl stretch), 3281(C-CH stretch) 1280, $1657(>\mathrm{C}=\mathrm{O}$ in conjugation with $\mathrm{C}=\mathrm{C}), 1594,1540(>\mathrm{C}=\mathrm{C}<$ in conjugation with $\mathrm{C}=\mathrm{O})$, $\left.{ }^{1} \mathrm{HNMR}\left(\mathrm{CDCl}_{3}\right), \delta(\mathrm{ppm}): 7\right): 7.047\left(\mathrm{~d}, 2 \mathrm{H}, \mathrm{Ar} 3^{\prime}, 5^{\prime} \mathrm{H}\right), 7.37$ (d, 2H, Ar 2',6'H), 7.58(d,1Ha, $\mathrm{J}=8.3 \mathrm{~Hz},), 7.30(\mathrm{~d}, 1 \mathrm{Hb}, \mathrm{J}=3.43 \mathrm{~Hz},=\mathrm{CH}), 8.03(\mathrm{~d}, 2 \mathrm{H}, \operatorname{Ar} 2 ", 6 "-\mathrm{H})$.

Compound 17: (2E)-3-(2-Furyl)-1-(3-hydroxyphenyl)-2-propen-1-one

M.pt $116-118{ }^{\circ} \mathrm{C}, \mathrm{MW}=232$, IR $(\mathrm{KBr}): 3281(\mathrm{C}-\mathrm{CH}$ stretch $) 1280,1657(>\mathrm{C}=\mathrm{O}$ in conjugation with $\mathrm{C}=\mathrm{C}), 1594,1540(>\mathrm{C}=\mathrm{C}<$ in conjugation with $\mathrm{C}=\mathrm{O}),{ }^{1} \mathrm{HNMR}\left(\mathrm{CDCl}_{3}\right), \delta$ (ppm): 7.047(d, 2H, Ar 3', 5'H), 7.37 (d, 2H, Ar 2',6'H), 7.58(d,1Ha, J = 8.3 Hz, ), 7.30(d, $1 \mathrm{Hb}, \mathrm{J}=3.43 \mathrm{~Hz},=\mathrm{CH}), 8.18$ (d, 2H, Ar 2",6"-H), H furan(d, 3.43, J=1.753).

\section{Anti-asthmatic activity}

Molecular modelling studies on the benzylidene-acetophenone suggest that the ortho hydroxy group substitution in the phenyl ring with heterocyclic ring have a potential of antiinflammatory activity. To keep this thing in mind, we synthesized the compounds 1 to 17 with modifications at positions R1 to R5 of the parent moiety (Table $1 \& 2$ ). The compound 8,13 and 17 from three different series were selected for the anti-inflammatory and bronchodilatory activities.

Table 3. Percentage response of selected compounds inhibiting histamine induced bronchospasm

\begin{tabular}{ccccc}
\hline \multirow{2}{*}{$\begin{array}{c}\text { Molar } \\
\text { concentrations }\end{array}$} & $\begin{array}{c}\text { Rolipram (Standard } \\
\text { Bronchodialtor) }\end{array}$ & Compound 8 & Compound 17 & Compound 13 \\
\cline { 2 - 5 } & - & 15.76 & 12.87 & 7.31 \\
15.5 & 38.2 & 18.32 & 16.71 & 10.79 \\
22.5 & 46.1 & 23.67 & 20.43 & 15.16 \\
30.1 & 53.8 & 32.59 & 29.95 & 18.42 \\
37.7 & 84.6 & 48.43 & 36.32 & 22.85 \\
45.3 & 92.0 & 59.80 & 41.43 & 24.35 \\
48.9 & 100 & --- & --- & -- \\
\hline
\end{tabular}

The anti-inflammatory activity was evaluated by using the rat paw edema model. In this model, the edema was induced by injection of a solution of carrageenan in saline into the hind footpad of mouse. This induces an acute swelling of the paw that becomes maximal $\sim 3 \mathrm{~h}$ after 
the injection. This model has long been used by researchers to assess the anti-inflammatory properties of agents such as nonsteroidal anti-inflammatory drugs (NSAIDs) that inhibit prostaglandin production. After the swelling reached at its maximum the different dosage of the selected compounds were injecte in the mouse, and the response of the swelling to these compound were measured by using Vernier Caliper (Mitutoyo 530-104).

The bronchodialtory activity was measured by using the mouse trachea. The mouse treachea was extracted and placed in saline. The bronchial constriction was measured before and after the injection of drugs. The similar measurements were repeated for the selected compounds.

All the three compounds were tested for the anti-inflammatory property, all of them showed moderate to good anti-inflammatory activity (Figure 2). To evaluate the bronchodilatory activity, rat tracheal strip was utilized and the percentage response produced by the compound was compared to Rolipram (PDE4 inhibitor with bronchodialatory action). Compound 8 showed relatively higher anti-inflammatory activity which can be attributed to the substitution of hydrophobic group and 3, 4-dialkoxy groups. Compound 8 showed a 59.8\% bronchodialatory response when compared to rolipram (100\% response), whereas compounds 17 and 13 showed marginal activity i.e. $41.43 \%$ and $24.35 \%$ of the standard drug.

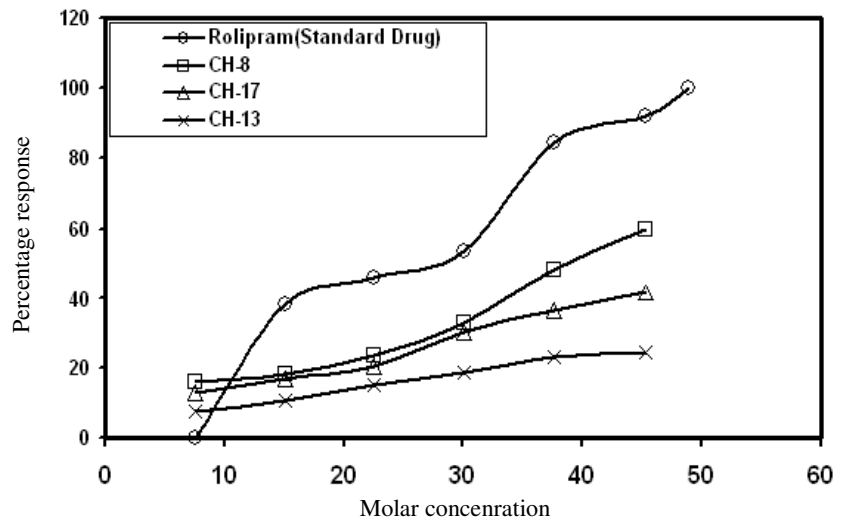

Figure 2. Percentage response of standard bronchodialatory drug (Rolipram) and selected compounds at different molar concentration

\section{Conclusion}

Various derivatives of the chalcone were synthesized and characterized. Three compounds were selected for the anti-inflammatory and bronchodilatory activity. Although, all compound shows low to moderate activity but, it was less than then the standard drugs. The mechanism of standard drugs are for anti asthmatic activity is known to reacted by the inhibition of phosphodiesterase 4 enzyme (PDE4). Although, these compounds shown promising anti asthmatic activity but the mechanism of their action is not clear. The PDE4 inhibition activity of compounds could open new molecular target for antiasthmatics drugs. However, from this study it was not clear that whether the compound possesses PDE4 inhibitor activity or simply they have the anti-inflammatory and bronchodilatory properties. This need to be investigated in future by the researchers. So, our study suggests that, although the benzylidene-acetophenone compounds used for the pharmacological activity were not as potent as rolipram, yet, this series of compounds have potential to provide template for further research. 


\section{Acknowledgment}

We want to thank Guru Jambheshwar University of Science and Technology for providing financial support for this research.

\section{References}

1. Won S J, Liu C T, Tsao L T, Ko H H, Wang J P and Lin C N, Eur J Med Chem., 2005, 40(1), 103-112; DOI:10.1016/j.ejmech.2004.09.006

2. Yu D C, Panfilova L V and Boreko E I, Pharm Chem., 1982, 16(2), 103-105; DOI:10.1007/BF00762027

3. Kamilah Hylton, Manuvesh Sangwan and Somenath Mitra, Analy Chim Acta, 2009, 653(1), 116-120; DOI:10.1016/j.aca.2009.08.042

4. $\quad$ Choudhary et al. Int J Pharm Pharm Sci., 2011, 3(3), 125-128.

5. Jeffrey J A, Pamela E O, Jared L R, Jeffrey N J, Peter D M, Linda M O, Pamela S W and Beth L E, Bioorg Med Chem Lett., 1996, 6(8), 995-998; DOI:10.1016/0960894X(96)00134-5

6. Manuvesh Sangwan and Lev N Krasnoperov, J Phys Chem A, 2013, 117(14), 29162923; DOI:10.1021/jp4000889

7. Lipworth B J, Lancet. 2005, 365(9454), 167-75; DOI:10.1016/S0140-6736(05)17708-3

8. Manuvesh Sangwan, Evgeni N Chesnokov and Lev N Krasnoperov, J Phys Chem A, 2012, 116(34), 8661-8670; DOI:10.1021/jp305070c

9. Kroegel C and Foerster M., Expert Opin Investig Drugs, 2007, 16(1), 109-24; DOI:10.1517/13543784.16.1.109

10. Horng H K, Lo T T, Kun L Y, Cheng T L, Jih P W and Chun N L, Bioorg Med Chem Lett., 2003, 11(1), 105-111; DOI:10.1016/S0968-0896(02)00312-7

11. Khatib S, Nerya O, Musa R, Shmuel M, Tamir S and Vaya J, Bioorg Med Chem., 2005; 13, 433-441; DOI:10.1016/j.bmc.2004.10.010

12. Chang Te, Int J Mol Sci., 2009; 10(6), 2440-2475; DOI:10.3390/ijms 10062440

13. Fabio S, Stefania B, Luca C, Gabriella V, Michele M and Luciano, A, Eur J Med Chem., 1998, 33(11), 859-866; DOI:10.1016/S0223-5234(99)80010-5

14. Manuvesh Sangwan and Lev N.Krasnoperov, J Phys Chem A, 2012, 116(48), $11817-$ 11822; DOI:10.1021/jp308885j

15. Manuvesh Sangwan, Evgeni N Chesnokov and Lev N Krasnoperov, J Phys Chem A, 2012, 116(24), 6282-6294; DOI:10.1021/jp211805v

16. Rao Y K, Fang S H and Tzeng Y M, Bioorg Med Chem., 2009; 17, 7909-7914; DOI:10.1016/j.bmc.2009.10.022

17. Lei Zhu, Manuvesh Sangwan, Li Huang, Juan Du and Liang T Chu, J Phys Chem A, 2015, 119(20), 4907-4914; DOI:10.1021/acs.jpca.5b00951

18. Ahmed M G, Romman U K R, Ahmed S M nad Akhter K, J Sci Ind Res., 2007, 42(1), 45-52.

19. Manuvesh Sangwan, Chao Yan, Evgeni N Chesnokov and Lev N Krasnoperov, $J$ Phys Chem A, 2015, 119(28), 7847-7857; DOI:10.1021/acs.jpca.5b01276 